\title{
Drug-induced interstitial lung disease (DILD) in molecular targeted therapy
}

\author{
Akihiko Gemma
}

Received: 26 October 2012/ Published online: 15 November 2012

(C) Japan Society of Clinical Oncology 2012

The development of molecularly targeted agents has been a key factor in recent advances in cancer therapy, and some of these agents are now considered standard therapies for various types of carcinoma. The toxicity of molecularly targeted agents is different from that of cytotoxic antitumor agents. ILD in Japanese patients treated with molecular targeting agents has been the focus of many studies.

Among tyrosine kinase inhibitors, gefitinib and erlotinib are associated with an increase in the incidence of ILD in Japanese patients. Gefitinib-induced DLI was reported to be $3.5 \%$ in a retrospective analysis and $5.8 \%$ in a prospective study of Japanese patients with non-small-cell lung cancer (NSCLC). In a cohort study including gefitinib and chemotherapy in Japanese patients with NSCLC, the naive cumulative incidence rates at the end of 12 -week follow-up were $4.0 \%$ for gefitinib versus $2.1 \%$ for conventional chemotherapy.

Little was known about drug-induced ILD when acute ILD-type events developed in Japanese patients treated with molecularly targeted agents including gefitinib. A better understanding of drug-induced ILD is required, including more reliable data about the incidence of events associated with different treatments and identification of the risk factors for this type of ILD. Recent advances in imaging, molecular examination, and pathology have been used in postmarketing surveillance studies designed and conducted by an independent academic team to define the risk and to increase the amount of evidence about ILD related to various molecularly targeted anticancer agents. The present analysis provides useful information about ILD to health-care professionals involved in treatment using molecular targeted therapy. These studies may shed light on the underlying mechanisms of drug-induced ILD and appropriate evidence-based strategies that can be used to prevent or manage these events. At this time, information about ILD by these molecular targeted agents including anti-EGFR antibodies, mTOR inhibitors, bortezomib, and multi-kinase inhibitors has accumulated. The difference in ILD according to causative drugs has been clarified. As for the treatment of DILD, the general rule is the discontinuation of the offending drug, and, if necessary, the administration of corticosteroids is indicated. However, exceptional treatment is required for DILD caused by mTOR inhibitor, for which we must consider adequate management. Based on this information, the guideline for drug-induced ILD was revised by the Japanese Respiratory Society this year.

In this issue, two experts describe the most recent findings from internal medicine and radiology in this field. We hope that these review articles will be helpful for understanding DILD in molecular targeted therapy.

Conflict of interest Akihiko Gemma is receiving a research grant from Pfizer Inc.; Akihiko Gemma has received lecture fees from Chugai Pharmaceutical Co., Ltd., Novartis Pharma K.K., Pfizer Inc., and Bayer Yakuhin, Ltd.
A. Gemma ( $\square)$

Division of Pulmonary Medicine and Oncology, Department of Internal Medicine, Graduate School of Medicine, Nippon Medical School,

1-1-5 Sendagi, Bunkyo-ku,

Tokyo 113-8603, Japan

e-mail: agemma@nms.ac.jp 